\title{
An Integral Sliding Mode-Based Robust Consensus Control Protocol Design for Electro-Mechanical Systems
}

\author{
Qudrat KHAN ${ }^{*}$, Rini AKMELIAWATI ${ }^{2}$, Mahmood Ashraf KHAN ${ }^{1}$ \\ ${ }^{1}$ CAST, COMSATS Institute of Information Technology, Islamabad, 44000, Pakistan \\ qudratullah@comsats.edu.pk (*Corresponding author), mahmoodashraf@comsats.edu.pk \\ ${ }^{2}$ Kulliyyah of Mechatronics Engg, International Islamic University, Kuala Lumpur, Malaysia \\ rakmelia@iium.edu.my
}

\begin{abstract}
This paper proposes a consensus tracking control for a class of second-order multi-agent nonlinear systems and generalizes the concept of integral sliding mode for networked systems. This design relies upon an integral manifold which is defined as a function of the consensus error variables. The designed integral manifold helps in the establishment of sliding mode without reaching phase. Consequently, the robustness against uncertainties is guaranteed from the very start. The continuous control components, of the control laws, governs the dynamics of the nonlinear system in sliding mode and the discontinuous terms handle the disturbances. The stability analysis is given to show the sliding mode establishment and an example is considered to demonstrate the benefits of the proposed strategy.
\end{abstract}

Keywords: Networked multi-agent systems, Uncertain systems, Integral sliding mode, Robust Control.

\section{Introduction}

Owing to the significant applications in rendezvous control of multi nonholonomic agents [8, 11, 14], formation control [2, 22], and flocking altitude alignment [3], the consensus control design of multi-agent systems has been the key focus of the researchers for almost two decades. These systems are either supposed to follow a specific pattern of motion or they are allowed to follow a leader which one may call leader consensus control. In the leader-follower systems, the leader influences the behaviour of the followers while remaining independent of the effects of the followers. Therefore, the control of the leader reflects the control of networks. This design strategy simplifies the overall implementation procedure of the control inputs and also prevents the energy loss with reduced cost [15]. But in practice, the availability of the leader's input to the followers may not be possible. For example, multiple missiles fired to follow an aircraft do not know about the aircraft's inputs and dynamics. Therefore, it is of prime importance to investigate control strategies which make use of little information related to the leader e.g., position and velocity availability to at least one follower of the network which must be capable to send information to a small portion of the network.

Recently, a number of papers have been focused on the cooperative regulation problem (consensus) and cooperative tracking problems (synchronization) of networked systems. In consensus the controllers are designed to drive all the networked agents/nodes to a consensus equilibrium which mainly depends on the initial conditions [16]. This is named consensus without leader. On the other hand, in synchronization the followers are supposed to follow the leader's output which is, in general, considered the as desired command for the followers (see for instance, $[17,18])$. The control algorithms, for consensus and the synchronization, need to be robust because, in practice, these systems operate under the action of disturbances. In the existing literature, a number of approaches, based on sliding mode theory [20], are developed for the robustness performance of uncertain nonlinear systems [1, 7]. Note that sliding-mode-based techniques were used for the consensus and synchronization control of multi-agent systems $[10,12,23]$. The synchronization problem posed in [12] provides finite-time consensus for second-order multi-robot systems whereas [23] has proposed neural network-based sliding mode control design for a class of higher-order feedback linearizable nonlinear systems. The strategies designed so far were based on the conventional sliding mode control (SMC) which is sensitive to a class of disturbances in the reaching phase. Therefore, in the past decades, the reaching phase free sliding mode control strategy development was the key focus of several researchers. In order to reject the matched uncertainties, an integral sliding mode-based strategies were proposed in $[9,13,21]$, whereas the mismatched uncertainties were suppressed via synergic strategies of an integral sliding mode and an $H_{\infty}$ control $[4,5$, 18]. Similarly, the other sliding-mode-based consensus and synchronization strategies provided robustness consensus or multi-robot systems. 
The main contribution, of this article, is that the concept of integral SMC is generalized to a network of nonlinear electro-mechanical systems instead of a single electro-mechanical system. Therefore, the structure of the control along with the stability analysis is entirely different from the existing literature. This design algorithm is different from that of [12], where a control input channel was fixed, while in the present article the authors have taken into account a state-dependent control input channel. The investigated control algorithm provides more benefits in terms of enhanced robustness with suppressed chattering and improved performance. Thus, the contribution in this article is substantial. The proposed control technique takes into account a well-defined problem. The problem formulation for the networked electro-mechanical systems with clear consensus scenarios is presented in Section 2. The control design for the posed problem, along with closed loop stability analysis, is addressed in Section 3. The efficacy of the newly developed control algorithm for this class is shown by the results of a numerical example in Section 4. In the end, the article is concluded, in Section 5, followed by the relevant references.

\section{Problem Formulation and Mathematical Preliminaries}

\subsection{Definitions}

A multi-agent system considered with a leader and $n$ followers which are also termed as nodes or vertices is denoted by $\mathcal{V}=\left\{\mathcal{V}_{0}, \mathcal{V}_{1}, \ldots, \mathcal{V}_{n}\right\}$, the set of edges is denoted by $\mathcal{E} \subseteq \mathcal{V} \times \mathcal{V}$ and a related graph is denoted by $\mathcal{G}=\{\mathcal{V}, \mathcal{E}\}$. Note that $\left(\mathcal{V}_{i}, \mathcal{V}_{j}\right) \subseteq \mathcal{E}$ if node $i$ is connected to node $j$. A direct path from node $i$ to $j$ is a sequence of successive edges in the form $\left\{\left(\mathcal{V}_{i}, \mathcal{V}_{k}\right),\left(\mathcal{V}_{k}, \mathcal{V}_{l}\right), \ldots,\left(\mathcal{V}_{m}, \mathcal{V}_{j}\right)\right\}$. In a directed graph the node $i$ can send information to node $j$ but not necessarily vice versa whereas in an undirected graph the two-way information is always possible (for more detail, see [19] and the references therein).

A weighted adjacency matrix $A=\left[a_{i j}\right] \in \mathfrak{R}^{n+1 \times n+1}$ of a graph $\mathcal{G}$ consists of nonnegative elements $a_{i j}>0$ if $\left(\mathcal{V}_{j}, \mathcal{V}_{i}\right) \in \mathcal{E}$ and $a_{i i}=0$ otherwise. In addition,

let $d_{i}=\sum_{j=0}^{n} a_{i j}, D=\operatorname{diag}\left\{d_{0}, d_{1}, \ldots, d_{n}\right\}$, and the Laplacian of weighted graph $\mathcal{G}$ is given by $L=D-A \in \mathfrak{R}^{n+1 \times n+1}$.

\subsection{Problem formulation}

In this work the dynamics of a leader system with the following state space realization is considered

$\dot{x}_{01}=x_{02}$

$\dot{x}_{02}=f_{0}\left(t, x_{0}\right)$

where $x_{0}=\left[x_{01}, x_{02}\right]^{T}$ represents the position and velocity vector of the leader system, $f_{0}\left(t, x_{0}\right)$ is the smooth scalar function which drives the leader. The dynamics of an $i^{\text {th }}$ follower is described via the subsequent state space realization

$\dot{x}_{i 1}=x_{i 2}, \quad i=1,2, \ldots, n$

$\dot{x}_{i 2}=f_{i}\left(x_{i}\right)+g_{i}\left(x_{i}\right) u_{i}+\Delta_{i}\left(x_{i}, t\right)$

where $x_{i}=\left[x_{i 1}, x_{i 2}\right]^{T}$ is the state vector of the $i^{\text {th }}$ follower. The term $\Delta_{i}\left(x_{i}, t\right)$ describes the matched uncertainties in the followers' dynamics and $u_{i}$ are the controlled inputs. In order to keep the controllability of each agent, it is assumed that $g_{i}\left(x_{i}\right) \neq 0$. Furthermore, it is also assumed here that

$\left\|\Delta_{i}\left(x_{i}, t\right)\right\| \leq C_{i}$

where $C_{i}$ are positive constants. The system considered here consists of $n+1$ agents. The leader's dynamics is made different from the follower dynamics by adding the subscript 0 . The remaining $n$ agents work as followers. A directed graph $\mathcal{G}=\{\mathcal{V}, \mathcal{E}\}$, with $\mathcal{V}=\left\{\mathcal{V}_{0}, \mathcal{V}_{1}, \ldots, \mathcal{V}_{n}\right\}$, is used to express the relationship between the leader and followers. The adjacency matrix is

$A_{i}=\left[\begin{array}{cccc}0 & 0 & \cdots & 0 \\ a_{10} & a_{11} & \cdots & a_{1 n} \\ a_{20} & a_{21} & \cdots & a_{2 n} \\ \vdots & \vdots & \ddots & \vdots \\ a_{n 0} & a_{n 1} & \cdots & a_{n n}\end{array}\right]$

A sub-graph $\overline{\mathcal{G}}=\{\overline{\mathcal{V}}, \overline{\mathcal{E}}\}$ denotes the topology of the $n$ followers. Now, owing to the followers' topology one may have

$\bar{A}_{i}=\left[\begin{array}{cccc}a_{11} & a_{12} & \cdots & a_{1 n} \\ a_{21} & a_{22} & \cdots & a_{2 n} \\ \vdots & \vdots & \ddots & \vdots \\ a_{n 1} & a_{\mathrm{n} 2} & \cdots & a_{n n}\end{array}\right]$ 
In addition, let $\bar{d}_{i}=\sum_{j=0}^{n} a_{i j}, \bar{D}=\operatorname{diag}\left\{\bar{d}_{1}, \ldots, \bar{d}_{n}\right\}$. Consequently, one has $\bar{L}=\bar{D}-\bar{A} \in \mathcal{R}^{n \times n}$. In this work $a_{i j}=1$ if $\left(\mathcal{V}_{j}, \mathcal{V}_{i}\right) \in \mathcal{E}$ and $a_{i j}=0$ otherwise. Next, $\bar{B}=\operatorname{diag}\left[\bar{b}_{1}, \ldots, \bar{b}_{n}\right]$, where $b_{i}=1$ if the agent $i$ is connected with the leader and otherwise 0 .

It is assumed that the function $f_{0}\left(t, x_{0}\right)$ is unknown to any follower. However, the upper bound of this function is known to its neighbours. It is also assumed that the leader's position $x_{01}$ and velocity $x_{02}$ is available to its neighbours. The problem (Problem-a) which the authors are going to address is that the $n$ followers should track the leader in the presence of uncertainties. To get a solution to this problem, it is reformulated by defining the consensus error variables in the forthcoming equations

$$
\begin{aligned}
& e_{i 1}=\sum_{j=1}^{n} a_{i j}\left(x_{i 1}-x_{j 1}\right)+b_{i}\left(x_{i 1}-x_{01}\right) \\
& e_{i 2}=\sum_{j=1}^{n} a_{i j}\left(x_{i 2}-x_{j 2}\right)+b_{i}\left(x_{i 2}-x_{02}\right)
\end{aligned}
$$

Owing to this definition, the error dynamics along the dynamics described by (1) and (2), appears as follows

$$
\begin{aligned}
\dot{\sum}_{1} & =\sum_{2} \\
\dot{\Sigma}_{2} & =(\bar{L}+\bar{B})(f(x)+g(x) u+\Delta(x, t)) \\
& -\overline{1} f_{0}(t, x)
\end{aligned}
$$

where

$$
\begin{aligned}
& \sum_{1}=\left[e_{11}, e_{21}, e_{31}, \ldots, e_{n 1}\right]^{T}, \\
& \sum_{2}=\left[e_{12}, e_{22}, e_{32}, \ldots, e_{n 2}\right]^{T},
\end{aligned}
$$$$
f(x)=\left[f_{1}\left(x_{1}\right), f_{2}\left(x_{2}\right), \ldots, f_{n}\left(x_{n}\right)\right]^{T},
$$$$
g(x)=\operatorname{diag}\left[g_{1}\left(x_{1}\right), g_{2}\left(x_{2}\right), \ldots, g_{n}\left(x_{n}\right)\right]
$$$$
u=\left[u_{1}, u_{2}, \ldots, u_{n}\right]^{T}
$$

and

$$
\Delta(t, x)=\left[\Delta_{1}\left(t, x_{1}\right), \ldots, \Delta_{n}\left(t, x_{n}\right)\right]^{T} .
$$

The new problem (Problem-b) is that of steering the states $\left[\sum_{1}, \sum_{2}\right]^{T}$, of the multi-input multi-output system, to the origin in the presence of matched uncertainties; that is, state regulation becomes the ultimate target. The solution to Problem-b is, consequently, the solution to Problem-a.

\section{Control Algorithm Design}

This section presents the integral sliding mode control design for the system developed in the aforementioned problem formulation. The control input is, generally, expressed as follows

$u=u_{a}+u_{b}$

where $u_{a}$ is the control input which governs the system in sliding mode and $u_{b}$ is the control input which effectively nullifies or diminishes the worst effects of matched disturbances. In the subsequent study, the design is systematically outlined.

\subsection{Integral manifold design}

The integral sliding manifold design for the $i^{\text {th }}$ system can be defined as follows

$\sigma_{i}=\lambda_{i} e_{i 1}+e_{i 2}+z_{i}$

The integral manifold for the overall system may be written as

$\sigma=\Lambda \sum_{1}+\left(\sum_{2}+Z\right)$

where $\Lambda=\operatorname{diag}\left[\lambda_{1}, \lambda_{2}, \ldots, \lambda_{n}\right] \in \mathfrak{R}^{n \times n}$ is the system's performance parameter matrix and $Z \in \mathfrak{R}^{n \times 1}$ is the vector of the integral terms of the integral manifolds.

\subsection{Design of $u_{a}$}

The system reported in (6), without perturbations, can be replaced as follows

$\dot{\Sigma}_{1}=\sum_{2}$

$\sum_{2}=\psi\left(\sum_{1}, \sum_{2}, u\right)+u$

where

$\psi\left(\sum_{1}, \sum_{2}, u\right)=(\bar{L}+\bar{B}) f(x)$

$+((\bar{L}+\bar{B}) g(x)-\overline{1}) u-\overline{1} f_{0}(t, x)$

It is suitable to assume that $\psi\left(\sum_{1}, \sum_{2}, u\right)=0$ at the very beginning. The validity of this assumption 
is authenticated by component $u_{a}$ at time $t=0$. Consequently, the system (10) becomes

$\dot{\Sigma}_{1}=\sum_{2}$

$\sum_{2}=u_{a}$

This system, for an $i^{\text {th }}$ follower, in matrix form can be written as

$\dot{E}_{i}=A_{i} E_{i}+B_{i} u_{a i}$

where $E_{i}=\left[\begin{array}{ll}e_{i 1} & e_{i 2}\end{array}\right]^{T}$ is the vector of the consensus errors, $A_{i}$ and $B_{i}$.are system distribution and input channel matrices and $u_{a i}$ is the stabilizing control input to the error dynamic system.

The emphasis here is on the development of the control design strategy and the authors consider the linear quadratic regulator (LQR) procedure suitable in this scenario. Therefore, the control component $u_{a i}$ will appear as follows

$u_{a i}=k_{i 1} e_{i 1}+k_{i 2} e_{i 2}=k_{i}^{T} E_{i}$

where $k_{i}=\left[k_{i 1} k_{i 2}\right]^{T}$ is the vector of the gains designed via the LQR procedure. Note that each component of this part of the control input minimizes the Quadratic cost function

$J_{i}=\frac{1}{2} \int_{0}^{\infty}\left(E_{i} Q_{i} E_{i}^{T}+u_{a i}^{T} R_{i} u_{a i} d t\right)$

subject to the dynamics reported in the aforesaid system. Note that the designer parameters of the aforesaid linear control component can be computed by solving the forthcoming algebraic Riccati equation

$A_{i}^{T} P_{i}+P_{i} A_{i}-P_{i} B_{i} R_{i}^{-1} B_{i}^{T} P_{i}+Q_{i}=0$

and

$k_{i}=-R_{i}^{T} B_{i} P_{i}$

where $P_{i}$ and $Q_{i}$ are symmetric and positive definite matrices. This control component will steer the error states to zero asymptotically.

\subsection{Design of $\mathbf{u}_{b}$}

The design of the current distributed control laws, for networked electro-mechanical systems, is analogous to that of [13]. The time derivative of the integral manifold along the overall error dynamic equation (6) becomes

$$
\begin{aligned}
& \dot{\sigma}=\Lambda \sum_{2}+(\bar{L}+\bar{B}) f(x) \\
& +((\bar{L}+\bar{B}) g(x)-\overline{1}) u \\
& -\overline{1} f_{0}(t, x)+u_{a}+u_{b}+(\bar{L}+\bar{B}) \Delta+\dot{Z}
\end{aligned}
$$

Choosing

$$
\dot{Z}=-u_{a}-\Lambda \sum_{2}
$$

One may have

$$
\begin{aligned}
& \dot{\sigma}=(\bar{L}+\bar{B}) f(x)+((\bar{L}+\bar{B}) g(x)-\overline{1}) u_{a} \\
& +(\bar{L}+\bar{B}) g(x) u_{b}-\overline{1} f_{0}(t, x)+(\bar{L}+\bar{B}) \Delta
\end{aligned}
$$

Now, the expression of $u_{b}$ can be chosen as follows

$$
\begin{aligned}
u_{b}= & -((\bar{L}+\bar{B}) g(x))^{-1} \\
& \times\left((\bar{L}+\bar{B}) f(x)+((\bar{L}+\bar{B}) g(x)-\overline{1}) u_{a}\right. \\
& +K \operatorname{sign}(\sigma)
\end{aligned}
$$

where $K$ is a vector of the gains $K_{i}$ of the discontinuous terms of $u_{b i}$ which are requird to enforce the sliding mode and $\operatorname{sign}(\sigma)=\left[\operatorname{sign}\left(\sigma_{1}\right) \operatorname{sign}\left(\sigma_{2}\right) \ldots \operatorname{sign}\left(\sigma_{n}\right)\right]$.The control signal of the $i^{\text {th }}$ node can be expressed via the subsequent mathematical formula

$$
\begin{aligned}
& u_{b i}=-\left(\left(\sum_{j=1, j \neq i}^{n} a_{i j}+b_{i}\right) g_{i}\left(x_{i}\right)\right)^{-1} \\
& \times\left(\begin{array}{l}
\left(\sum_{j=1, j \neq i}^{n} a_{i j}+b_{i}\right) f_{i}\left(x_{i}\right)+\left(\left(\sum_{j=1, j \neq i}^{n} a_{i j}+b_{i}\right) g_{i}\left(x_{i}\right)-1\right) u_{a i} \\
+K_{i} \operatorname{sign}\left(\sigma_{i}\right)
\end{array}\right)
\end{aligned}
$$

\subsection{Stability analysis}

The stability analysis of the closed loop dynamics of the networked agent is carried out by considering a suitable Lyapunov function $V=\frac{1}{2} \sigma^{2}$ whose time derivative along the dynamics of (12) becomes

$$
\begin{aligned}
& \dot{V}=\sigma\left(\Lambda \sum_{2}+(\bar{L}+\bar{B}) f(x)\right. \\
& +((\bar{L}+\bar{B}) g(x)-\overline{1}) u-\overline{1} f_{0}(t, x) \\
& \left.+u_{a}+u_{b}+(\bar{L}+\bar{B}) \Delta+\dot{Z}\right)
\end{aligned}
$$


Making use of the -dynamic equation, reported in (13), one has

$$
\begin{aligned}
& \dot{V}=\sigma(\bar{L}+\bar{B}) f(x)+((\bar{L}+\bar{B}) g(x)-\overline{1}) u_{a} \\
& \left.+(\bar{L}+\bar{B}) g(x) u_{b}-\overline{1} f_{0}(t, x)+(\bar{L}+\bar{B}) \Delta\right)
\end{aligned}
$$

Now, substituting the value of the control input, one gets

$$
\dot{V}=\sigma\left((\bar{L}+\bar{B}) \Delta-K \operatorname{sign}(\sigma)-\overline{1} f_{0}(t, x)\right)
$$

or

$$
\dot{V} \leq-\eta \sqrt{V}
$$

if one has

$$
\min \left(K_{i}\right) \geq\|(\bar{L}+\bar{B}) \Delta\|+\left\|\overline{1} f_{0}(t, x)\right\|
$$

This confirms the enforcement of the sliding mode along the defined integral manifold from the initial time instant i.e. $\sigma=0$ at $t=0$ and all future times $t>0$.

Remark 1. In general, the practical systems are equipped only with position measurement sensor. Therefore, the velocities, required in the proposed strategy, can be estimated via the velocity observer [6].

\section{Illustrative example}

In this section, the design algorithm presented for the consensus control of the networks of nodes/agents in the presence of disturbances, is simulated for four followers and one leader. Both the leader and the followers are represented by second-order dynamic equations. The control objective is that the followers should track the leader in the presence of matched perturbations.

\subsection{Dynamic models of the leader and followers}

The dynamics of the leader is described by the following state space equations

$\dot{x}_{01}=x_{02}$

$\dot{x}_{02}=f_{0}\left(x_{01}, x_{02}, t\right)$

where $f_{0}\left(x_{01}, x_{02}, t\right)=-\frac{\sin \left(x_{01}\right)}{1+\exp (-t)}$ is the input to the leader's system. Meanwhile, the followers' dynamics is represented by the following systems $\dot{x}_{i 1}=\dot{x}_{i 2}, \quad i=1,2,3,4$

$\dot{x}_{i 2}=f_{i}\left(x_{i}\right)+g_{i}\left(x_{i}\right) u_{i}+\Delta_{i}\left(x_{i}, t\right)$

where $x_{i}=\left[x_{i 1}, x_{i 2}\right]^{T}$ is the state vector of the $i^{\text {th }}$ follower. The term $\Delta_{i}\left(x_{i}, t\right)=0.5 \sin (t)$ describes the matched uncertainties in the $f_{i}\left(x_{i}\right)=-\frac{g}{l} \sin \left(x_{i 1}\right)$ and $g_{i}\left(x_{i}\right)=\frac{1}{m l^{2}}$. The terms are system distribution and input channel functions such that $m, l$ and $g$ represents the mass, length and gravitational acceleration of a pendulum. The topology of these multi-agents is represented by a directed graph in Figure 1. $u_{i}, i=1,2,3,4$ are the controlled inputs to their respective systems. The adjacency, Laplacian and the interconnection relationship matrices, while keeping in view the directed graph in Figure 1, are described as follows

$\bar{A}=\left[\begin{array}{llll}0 & 1 & 1 & 1 \\ 0 & 0 & 1 & 0 \\ 0 & 1 & 0 & 0 \\ 0 & 0 & 0 & 0\end{array}\right]$

$\bar{D}=\operatorname{diag}\left[\begin{array}{llll}3 & 1 & 1 & 0\end{array}\right]$

The matrix is calculated via $\bar{L}=\bar{D}-\bar{A}$.

$\bar{L}=\left[\begin{array}{cccc}3 & -1 & -1 & -1 \\ 0 & 1 & -1 & 0 \\ 0 & -1 & 1 & 0 \\ 0 & 0 & 0 & 0\end{array}\right]$

and

$\bar{B}=\operatorname{diag}\left[\begin{array}{llll}0 & 0 & 1 & 1\end{array}\right]$

\subsection{Controller design}

The proposed design methodology is employed, in this section, to achieve the aforementioned task. The sliding variables are defined as follows

$e_{i 1}=\sum_{j=1}^{4} a_{i j}\left(x_{i 1}-x_{j 1}\right)+b_{i}\left(x_{i 1}-x_{01}\right)$

$e_{i 2}=\sum_{j=1}^{4} a_{i j}\left(x_{i 2}-x_{j 2}\right)+b_{i}\left(x_{i 2}-x_{02}\right)$

The integral manifold for the overall system may be written as

$\sigma=\Lambda \sum_{1}+\left(\sum_{2}+Z\right)$

where $\Lambda=\operatorname{diag}\left[\lambda_{1}, \lambda_{2}, \ldots, \lambda_{4}\right] \in \mathfrak{R}^{4 \times 4}$ and $Z \in \mathfrak{R}^{4 \times 1}$. The $\lambda_{i}, i=1,2,3,4$ are the performance parameters of the control component which drive the system from initial time instant. The final expressions of the controllers can be computed according to the aforesaid study. 


\subsection{Simulation Results}

The aforementioned network of multi-agent system is simulated while considering the controlled input designed according to the outlined strategy. The performance and robustness evaluation of this newly proposed controller is carried out in the presence of control input disturbances. In Figure 2 , the consensus tracking of the given network with the defined topology is displayed which shows the robust performance, from the very beginning, in the presence of disturbances. The corresponding error convergence of the followers is shown in Figure 3 which, once again, confirms the robust performance of the proposed controller. Furthermore, the integral manifolds' convergence is witnessed by Figure 4 and, consequently, authenticates the reaching phase elimination and robustness enhancement. The control inputs to each node are depicted in Figure 5. It is apparent from the results that the applied input is actuating the system with suppressed chatter. Hence, having looked at these results, one can conclude that integral sliding mode approach is a suitable candidate for the consensus control of a multiagent systems with input disturbances.

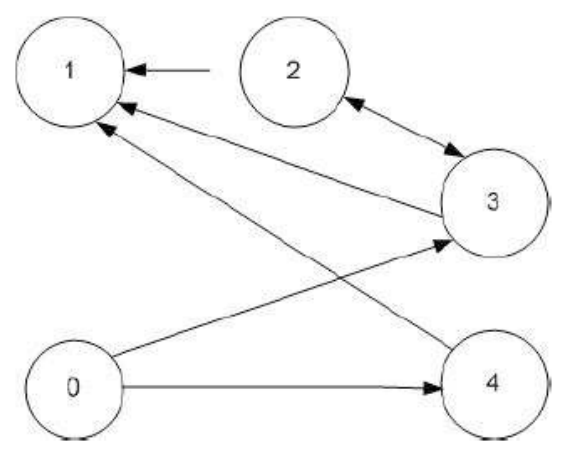

Figure 1. Topology of the network of four followers and a leader

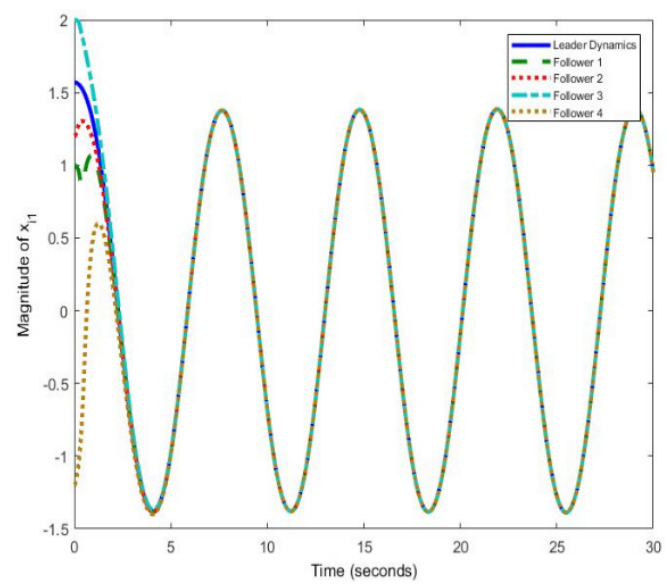

Figure 2. Position tracking of the leader by four followers

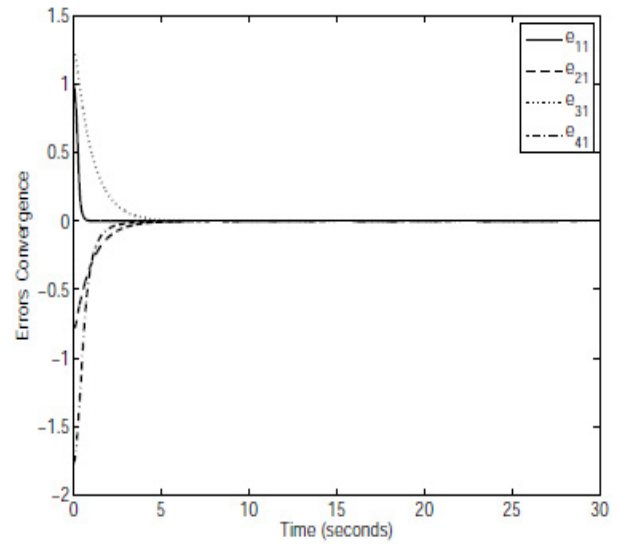

Figure 3. Errors' convergence of the four followers

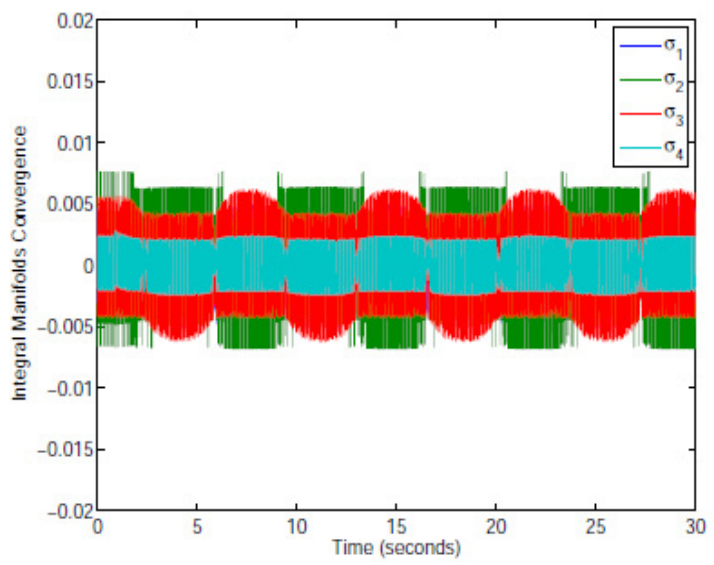

Figure 4. Integral manifolds of the four followers

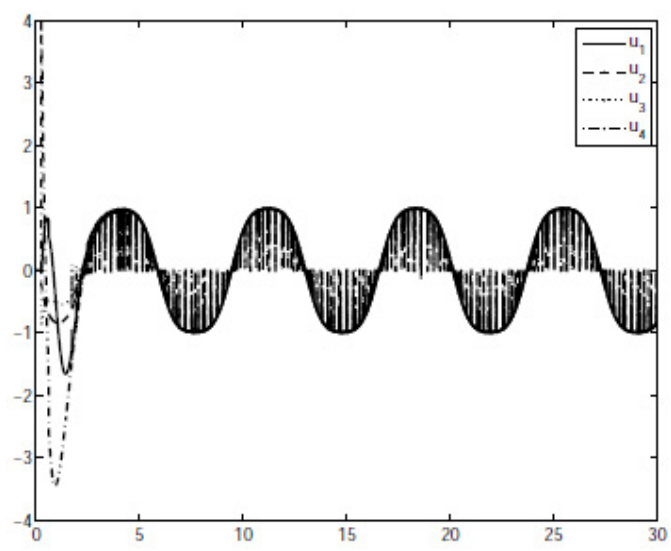

Figure 5. Control inputs for the consensus tracking control

\section{Conclusions}

This paper presented the consensus tracking control of multi-agent system described by a class of second-order uncertain nonlinear systems. The design relies upon a transformed system of errors' dynamics with control input uncertainties. An integral manifold based strategy is proposed for 
the aforesaid system. This strategy, as far as its definition is concerned, ensures the sliding mode from the very beginning which, consequently, results in enhanced robustness against input disturbances. The error dynamics regulation is confirmed via the LQR-based designed control

\section{REFERENCES}

1. Bartoszewicz, A. \& Latosinski, P. (2016). Sliding Mode Congestion Controller for Data Transmission Networks with Unknown and Variable Packet Loss Rates, Studies in Informatics and Control, 25(1), 109-121.

2. Bliman, P. A. \& Giancarlo, F. T. (2008). Average Consensus Problems in Networks of Agents with Delayed Communications, Automatica, 44(8), 1985-1995.

3. Castanos, F. \& Fridman, L. (2006). Analysis and Design of Integral Sliding Manifolds for Systems with Unmatched Perturbations, IEEE Transactions on Automatic Control, 51(5), 853-858.

4. Chang, J. L. (2009). Dynamic Output Integral Sliding Mode Control with Disturbance Attenuation, IEEE Transactions on Automatic Control, 54(11), 2653-2658.

5. Chihi, A., Ben-Azza, H., Jemli, M. \& Sellami, A. (2017). Nonlinear Discrete-Time Integral Sliding Mode Control of an Induction Motor: Real-Time Implementation, Studies in Informatics and Control, 26(1), 23-32.

6. Cruz-Zavala, E., Moreno, J. A. \& Fridman, L. (2011). Uniform Robust Exact Differentiator, IEEE Transactions on Automatic Control, 56(11), 2727-2733.

7. Cucker, F. \& Smale, S. (2007). Emergent Behavior in Flocks, IEEE Transactions on Automatic Control, 52(5), 852-862.

8. Dimarogonas, D. V. \& Kyriakopoulos, K. J. (2007). On the Rendezvous Problem for Multiple Nonholonomic Agents, IEEE Transactions on Automatic Control, 52(5), 916-922.

9. Edwards, C. \& Spurgeon, S. K. (1998). Sliding Modes Control: Theory and Applications. London, UK: Taylor and Francis.

10. El-Ferik, S., Qureshi, A. \& Lewis, F. L. (2014). Neuro-adaptive Cooperative component. An illustrative example, consisting of four followers and a leader, is considered in the end to demonstrate the effectiveness of this control candidate for a network of multi-agents subject to input disturbances.

Tracking Control of Unknown Higher Order Affine Nonlinear Systems, Automatica, 50(3), 798-808.

11. Huang, J., Farritor, S. M., Qadi, A. \& Goddard, S. (2006). Localization and Follow-theLeader Control of a Heterogeneous Group of Mobile Robots, IEEE/ASME Transactions on Mechatronics, 11(2), 205-215.

12. Khan, Q., Bhatti, A. I., Iqbal, S. \& Iqbal, M. (2011). Dynamic Integral Sliding Mode Control of Uncertain MIMO Nonlinear Systems, International Journal of Control Automation and Systems, 9(1), 151-160.

13. Khoo, S., Xie, L. \& Man, Z. (2009). Robust Finite Time Consensus Tracking Algorithm for Multirobot Systems, IEEE/ASME Transactions on Mechatronics, 14(2), 219-228.

14. Luo, R.C.\& Chen, T.M.(2000). Development of A Multi-behavior Based Mobile Robot for Remote Supervisory Control Through the Internet, IEEE/ASME Transactions on Mechatronics, 5(4), 376-385.

15. Ma, C. Q., Li, T. \& Zhang, J. F. (2008). Leader Following Consensus Control for Multi-agent Systems Under Measurement Noises. In Proceeding of $17^{\text {th }}$ World Congress International Federation on Automatic Control (pp. 1528-1533).

16. Ren, W. \& Beard, R. W. (2007). Distributed Consensus in Multi-Vehicle Cooperative Control. New York: Springer-Verlag.

17. Ren, W., Beard, R. \& Atkins, E. (2007). Information Consensus in Multivehicle Cooperative Control, IEEE Control System Magazine, 27(2), 71-82.

18. Rubagotti, M., Estrada, A., Castanos, F., Ferrara, A. \& Fridman, L. (2011). Integral Sliding Mode Control for Nonlinear Systems with Matched and Unmatched Perturbations, IEEE Transactions on Automatic Control, 56(11), 2699-2704. 
19. Saber, R.O.,Fax, J.A. \& Murray, R. M. (2007). Consensus and Cooperation in Networked Multi-agent Systems, Proceedings of IEEE, 95(1), 215-233.

20. Utkin, V. I. (1992). Sliding Modes in Control Optimization. Berlin, Germany: Springer-Verlag.

21. Utkin, V. I., Guldner, J. \& Shi, J. (1999). Sliding Mode Control in Electromechanical Systems. UK: Taylor and Francis.
22. Yang, E. \& Gu, D. (2007). Nonlinear Formation-Keeping and Mooring Control of Multiple Autonomous Underwater Vehicles, IEEE/ASME Transactions on Mechatronics, 12(2), 205-215.

23. Zhang, H. \& Lewis, F. L. (2012). Adaptive Cooperative Tracking Control of Higher Order Nonlinear Systems with Unknown Dynamics, Automatica, 48, 1432-1439. 